INRA Prod. Anim., 2004, 17 (3), 217-225
P. FONTAINE ${ }^{1}$, P.-Y. LE BAIL ${ }^{2}$

${ }^{1}$ INRA-INPL-UHP Nancy 1, Laboratoire de Sciences Animales, MAN, 34 rue Sainte Catherine, F-54000 Nancy

Courriel :

pascal.fontaine@lsa-man.uhp-nancy.fr

2 INRA, Station Commune de Recherche en Ichtyophysiologie, Biodiversité et Environnement, Campus de Beaulieu, F-35042 Rennes Cedex

Courriel :

Pierre-Yves.Lebail@beaulieu.rennes.inra.fr

\title{
Domestication et croissance chez les poissons
}

Quel que soit le débouché visé, la recherche de performances de croissance élevées est toujours un objectif pour les éleveurs, même si ce n'est pas un objectif toujours prioritaire. Les conséquences économiques liées à un faible taux de croissance concernent tout type d'élevage. De ce fait, la mise en place d'une démarche de domestication et la production de nouvelles espèces doivent prendre en compte les potentialités de croissance des espèces et ses limites. Regrouper les espèces à partir de caractéristiques communes des cycles de développement doit permettre de mieux appréhender les enjeux de la domestication par rapport à la nécessité de maîtriser et d'optimiser la croissance dans les élevages.

Actuellement, on considère qu'il existe environ 30000 espèces de poissons, en incluant celles a priori pas encore connues. Ce grand nombre d'espèces exprime une très forte variabilité phénotypique (forme, couleur, taille...). A titre d'exemple, l'ichtyodiversité guyanaise (eaux douce et saumâtre) présente $36 \%$ d'espèces de très petite taille (de 0 à $10 \mathrm{~cm}$ ), $26 \%$ d'espèces de petite taille (de 10 à $20 \mathrm{~cm}$ ), $14 \%$ d'espèces de taille moyenne (de 20 à $30 \mathrm{~cm}$ ) et $24 \%$ d'espèces de grande taille (plus de $30 \mathrm{~cm}$ ) (Planquette et al

\section{Résumé}

En s'appuyant sur les deux principales phases successives, la larviculture et le grossissement, qui caractérisent un cycle de production en aquaculture, la fonction de croissance est analysée sous l'angle d'une typologie fonctionnelle. Pour tous les stades de développement, une très forte hétérogénéité de croissance (intra- et interspécifique) est constatée, elle s'explique principalement par l'action de facteurs inhérents à l'espèce ou imposés (abiotiques et biotiques). Pour la phase de grossissement, cette forte variabilité phénotypique ne permet pas le regroupement d'espèces par rapport à des types de croissance donnés. De fortes interactions avec d'autres fonctions biologiques, comme la reproduction, contribuent à cet effet. La fonction de croissance ne permet pas de discriminer des groupes d'espèces en relation avec des stratégies fonctionnelles. Pour la phase de larviculture (stades larve, post-larve et juvénile), l'hétérogénéité de croissance intraspécifique et l'impact du cannibalisme pourraient être liés aux stratégies de vie des espèces de poissons.
1996, Keith et al 2000, Le Bail et al 2000). Au stade adulte, les poissons téléostéens peuvent donc présenter des tailles extrêmes d'une espèce à l'autre (moins de $2 \mathrm{~cm}$ pour le genre Fluviphylax, famille des poecillidés ; plus de $460 \mathrm{~cm}$ pour le genre Thunnus, famille des Thonnidés). Même s'il existe une très forte corrélation entre la taille maximale des espèces et leur vitesse de croissance dans un même biotope, l'indicateur «taille » ne peut être considéré seul dans une démarche de domestication, car d'autres caractéristiques biologiques majeures liées à (longévité, croissance ...) ou interagissant avec ce facteur (maturité sexuelle ...) jouent également un rôle important dans une telle démarche.

En aquaculture, lors de la domestication de nouvelles espèces, les enjeux de la croissance et de sa maîtrise diffèrent selon le stade de développement étudié. En larviculture (stades larvaire, post-larvaire et juvénile jusqu'à un poids moyen de quelques grammes), l'objectif majeur réside plus dans la régulation de l'hétérogénéité de croissance et du cannibalisme induit par une forte hétérogénéité de taille, que dans l'optimisation des performances de croissance proprement dites. Le cannibalisme, qui peut être une cause de mortalité majeure en larviculture, 
affecte souvent de manière très significative les taux de survie (Baras 1998, Fontaine 2004). En phase de pré-grossissement ou de grossissement, une optimisation des performances de croissance, associée à un contrôle de la qualité des produits, demeure souvent l'enjeu principal lorsqu'une démarche pour la domestication d'une nouvelle espèce est engagée.

\section{1 / Hétérogénéité de croissance, cannibalisme et domestication en larviculture}

Les variations de croissance, observées chez les poissons au cours des premiers stades de développement, résultent souvent d'une interaction d'un grand nombre de facteurs. Huston et De Angelis (1987) distinguent les facteurs inhérents à l'espèce et ceux imposés par les conditions d'élevage. Parmi ces deux types de facteurs, inhérents ou imposés, ils différencient également deux catégories, les facteurs interactifs (liés aux interactions sociales) et non interactifs. Ces regroupements, présentés dans le tableau 1 , ont été repris et adaptés par Kestemont et al (2003). Un même facteur, comme la disponibilité en aliment, peut agir de manière non interactive (hétérogénéité du milieu d'élevage) ou interactive (effet sur la croissance induit par la compétition alimentaire).

\section{1 / Rôle des facteurs inhérents à l'espèce}

Même si une espèce donnée présente une forte variabilité de croissance, cette dernière reste dans une gamme donnée et peut se référer à une courbe de croissance type, ce qui traduit le rôle des facteurs génétiques dans le contrôle de la croissance (Wotton 1999). Létude des populations de capucette de l'atlantique (Menidia menidia) le long de la côte atlantique d'Amérique du Nord illustre bien le rôle des facteurs génétiques (Conover 1992). En conditions expérimentales et à une température donnée avec une alimentation distribuée à satiété, les larves des populations septentrionales montrent une croissance supérieure par comparaison à celle des populations méridionales. Cette différence existe même si la température de l'eau est élevée et proche de celles rencontrées par les populations méridionales de capucette en milieu naturel, traduisant ainsi la notion de contregradient (Schultz et al 1996). L'origine génétique de cette différence de croissance a été confirmée par l'élevage de larves de capucette obtenues à partir de stocks de géniteurs élevés en conditions contrôlées. Plus récemment, une comparaison des performances de croissance de la perche commune Perca fluviatilis au cours des premiers stades de développement (en conditions de laboratoire), chez des populations de diverses origines géographiques (sud de l'Italie, sud-est et nordest de la France, Belgique), montre également d'importantes différences de croissance en fonction de l'origine géographique des larves (Mandiki et al 2004) : la vitesse de croissance est significativement plus élevée chez les populations d'origine septentrionale.

Au cours d'un cycle de développement, des changements morpho-anatomiques ou morpho-physiologiques influencent également les caractéristiques de croissance des jeunes poissons. Les métamorphoses (Pleuronectiformes), les changements importants de formes (de la larve au juvénile) et de milieu (espèces migratrices comme les anguillidés), la taille de l'ouverture buccale, l'inflation de la vessie gazeuse et l'ontogénie du tube digestif (mise en place et évolution de l'équipement enzymatique digestif, Cahu 2004) affectent directement la croissance aux premiers stades de développement (Ricker 1979, Dabrowski et al 1993). Logiquement, la taille des larves à l'éclosion joue aussi un rôle majeur, notamment au travers de l'importance des réserves vitellines qui vont agir sur l'âge (et la taille) au passage de l'endotrophie à l'exotrophie. D'une manière générale, les

Tableau 1. Mécanismes biologiques responsables des variations de taille et de perte de croissance chez les larves de poissons à nageoires en conditions d'élevage (Huston et De Angelis 1987, Kestemont et al 2003).

\begin{tabular}{|c|c|c|}
\hline & Non interactifs & Interactifs \\
\hline Inhérents & $\begin{array}{l}\text { Variations génétiques } \\
\text { Changements morpho-physiologiques }\end{array}$ & Changements morpho-physiologiques \\
\hline \multirow[t]{3}{*}{ Imposés } & $\begin{array}{l}\text { Hétérogénéité temporelle } \\
\text { Moment d'éclosion } \\
\text { Température } \\
\text { Durée du jour } \\
\text { Hétérogénéité de l'espace } \\
\text { Ressources abiotiques }\end{array}$ & $\begin{array}{l}\text { Compétition } \\
\text { Longueur du jour et intensité lumineuse } \\
\text { Turbidité } \\
\text { Disponibilité en aliment } \\
\text { Densité de stockage } \\
\text { Variation de taille initiale }\end{array}$ \\
\hline & $\begin{array}{l}\text { Taille des aliments } \\
\text { Disponibilité en aliment } \\
\text { Température } \\
\text { Clarté de l'eau }\end{array}$ & \multirow[t]{2}{*}{$\begin{array}{l}\text { Mortalité } \\
\text { Cannibalisme } \\
\text { Densité }\end{array}$} \\
\hline & $\begin{array}{l}\text { Mortalité } \\
\text { Densité }\end{array}$ & \\
\hline
\end{tabular}


changements de régime alimentaire, par exemple le passage d'un régime planctonophage à un régime ichthyophage, influencent fortement la croissance des jeunes poissons (Wotton 1999).

Selon ce dernier auteur, l'ensemble des facteurs inhérents détermine le potentiel de croissance des espèces. Toutefois, les croissances, observées d'une année sur l'autre dans un environnement donné ou dans différents environnements, varient très fortement chez une espèce donnée et aboutissent à une forte variabilité phénotypique. Cette plasticité ou flexibilité s'explique principalement par l'action de facteurs abiotiques (environnement physico-chimique, trophique...) ou biotiques (environnement populationnel) qui module l'expression du potentiel de croissance.

\section{2 / Rôle des facteurs imposés}

\section{a / Facteurs abiotiques}

Les poissons étant des animaux poïkilothermes, la température de l'eau affecte évidemment la croissance des larves de poissons en fonction notamment de son éloignement ou non de la zone de préférence thermique. Ce facteur a des effets sur l'hétérogénéité de croissance observée dans les élevages. Par exemple, une élévation de la température de 17 à $23^{\circ} \mathrm{C}$ s'accompagne d'une augmentation de l'hétérogénéité de taille des juvéniles de perche commune et, en conséquence, du taux de cannibalisme (Kestemont et Mélard 2000). De même, les facteurs d'éclairement (durée de la photopériode, intensité lumineuse, modalités des phases de transition jour/nuit...), en étroite relation avec la couleur des enceintes d'élevage et la gestion du nourrissage, agissent fortement sur la croissance aux premiers stades de développement. Une étude comparative conduite chez deux espèces de poissons piscivores, la perche commune et le bar Dicentrarchus labrax, montre que ces facteurs ont une action très variable selon le stade de développement concerné (Kestemont et al 2003). Les sensibilités inverses des larves et des postlarves sont largement liées à l'ontogenèse du système rétinien : faible densité de cônes simples, absence de doublets de cônes et pas de mosaïque chez les larves (Bone et al 1996). En fonction des caractéristiques comportementales et/ou écologiques des espèces, les effets des facteurs d'éclairement, qui agissent principalement sur l'activité, notamment alimentaire, des jeunes poissons, diffèrent (espèces à activité diurne, nocturne, crépusculaire ou mixte ; voir la synthèse bibliographique de Jourdan 1999). De nombreux autres facteurs physico-chimiques, comme la turbidité, le courant, la minéralisation, les teneurs en substances toxiques (ammoniac, nitrites) ... peuvent également moduler le développement des larves, post-larves et juvéniles. Ainsi, chez certaines espèces, une augmentation de la turbidité de l'eau permet une optimisation de la croissance en atténuant la lumière ambiante et une régulation du cannibalisme (Naas et al 1996, Rieger et Summerfelt 1997).
L'environnement trophique (disponibilité dans le temps et l'espace, aspects qualitatifs et quantitatifs), en interaction avec les facteurs environnementaux, joue également un rôle majeur dans l'expression du potentiel de croissance et la régulation de son hétérogénéité (Kestemont et al 2003). Les facteurs nutritionnels (composition de l'aliment, taux de rationnement, nombre de repas/jour, taille des particules alimentaires, moment du premier repas ...) sont principalement responsables de l'établissement de hiérarchies sociales et du cannibalisme dans les populations de jeunes poissons mises en élevage (Hecht et Pienaar 1993). La période de transition alimentaire que représente le sevrage des post-larves est une période particulièrement sensible, surtout chez les espèces piscivores, qui peut favoriser un accroissement de l'hétérogénéité de taille. L'importance du régime alimentaire (type de proies vivantes distribuées) sur l'évolution de l'hétérogénéité de poids a récemment été démontrée chez un characiforme tropical sud-américain, le Dorada Brycon moorei (Baras et Jobling 2002).

\section{b / Facteurs biotiques}

Les facteurs biotiques qui interviennent dans la régulation du potentiel de croissance des premiers stades de développement correspondent principalement à des variables populationnelles (densité, hétérogénéité initiale de taille, variabilité inter-individuelle des potentiels de croissance ...). Les effets de la densité d'élevage diffèrent selon les espèces et le stade de développement. Chez la perche commune, une diminution de la densité d'élevage de 100 à 10 larves/l accroît le taux de cannibalisme et réduit la survie chez les larves, alors que, pour les post-larves, une élévation de la densité de 1 à 10 individus/l diminue la survie et élève le taux de cannibalisme (Kestemont et al 2003). Chez le bar, des résultats similaires sont observés lors d'une élévation de la densité des post-larves de 5 à 20 individus/l. L'interprétation des effets de la densité doit considérer l'existence de comportements spécifiques, tels que la structuration en banc des juvéniles observée chez certaines espèces. Pour ce qui concerne le rôle des autres variables populationnelles, il a récemment été montré que le poids moyen initial et l'hétérogénéité initiale de poids des postlarves influencent significativement la croissance et la survie des cohortes élevées (Babiak et al 2004). En fait, les facteurs biotiques jouent un rôle majeur dans l'expression du cannibalisme en larviculture puisque le moment où apparaît ce phénomène et l'intensité de son impact dépendent fortement du rapport entre la taille du prédateur et celle de la proie d'une part et de l'âge des post-larves d'autre part (dès le premier jour post-éclosion chez Brycon moorei et à J30 chez le poissonchat africain Clarias gariepinus, Baras et Jobling 2002). L'émergence du cannibalisme est plus tardive chez les espèces marines qui présentent généralement de plus importants changements morpho-anatomiques. 


\section{3 / Stratégie de vie et domesti- cation en larviculture}

A notre connaissance, aucune réflexion scientifique n'a porté, à ce jour, sur la construction d'une démarche de domestication considérant les stratégies de vie des espèces et les mécanismes de régulation de la croissance pour les premiers stades de développement. Cependant, des éléments de la littérature laissent envisager de réelles perspectives. Une première piste réside dans les liens qui pourraient exister entre les stratégies reproductives des espèces et d'éventuels mécanismes de régulation de la qualité des larves. Par exemple, il a été récemment démontré, chez la perche commune, qu'au sein d'une même ponte, les larves ne possèdent pas les mêmes aptitudes à la croissance et la survie en fonction du moment d'éclosion (éclosion sur 5 jours). Les premières larves écloses, bien que significativement plus petites en poids, présentent des taux de survie et de croissance nettement supérieurs, tant en conditions d'élevage de type unimodal que multimodal (Kestemont et al 2003). De plus, leur croissance reste très homogène avec un coefficient de variation pondéral qui reste constant au cours des 3 semaines d'élevage (J1-J21). Considérant les principales caractéristiques écologiques de l'espèce (très féconde, piscivore, sujette au nanisme et au cannibalisme, pionnière dans la colonisation de nouveaux habitats ...), une meilleure « qualité » des premières larves écloses semble en accord avec la stratégie de vie de l'espèce. En effet, les cohortes de perche se trouvent souvent en surdensité dans un milieu oligotrophe ou mésotrophe (lacs alpins), aussi, en favorisant le développement de quelques individus $(20 \%)$ au détriment du reste de la cohorte $(80 \%)$, la pérennité de l'espèce semble mieux assurée. De plus, les larves tardives pourraient constituer une réserve alimentaire potentielle, le cannibalisme étant considéré par certains auteurs comme une stratégie adaptative chez les espèces omnivores ou piscivores (Nishimura et Hoshino 1999). Très peu de cannibalisme est observé chez les espèces ayant des stratégies de reproduction de type $\mathrm{k}$ (espèces peu fécondes, soins parentaux). En conséquence, il pourrait y avoir un lien entre les stratégies de vie des poissons (reproduction, type d'habitat, régime alimentaire ...) et les mécanismes biologiques régulant l'hétérogénéité de croissance et le cannibalisme lors des premiers stades de développement. Il faudrait également considérer la variabilité intraspécifique initiale (œufs, larves) de taille ou de poids qui peut varier fortement d'une espèce à l'autre (Bagenal 1971, Kamler 1992).

Regrouper les espèces selon une typologie fonctionnelle (surtout ciblée sur l'hétérogénéité de croissance et ses modes de régulation), en intégrant les critères majeurs caractérisant certains traits de vie (données biométriques, morpho-anatomiques, comportementales et écologiques) semble être une perspective très prometteuse pour mieux appréhender les problèmes posés par la domestication d'une nouvelle espèce. Y-a-t-il un véritable contrôle de la qualité des larves ?
Si oui, les mécanismes impliqués diffèrent-ils selon les différentes stratégies de vie des espèces ? La constitution d'une typologie doit permettre ultérieurement la mise en place d'études de biologie comparée.

\section{2 / Croissance et domestication en phase de grossissement}

\section{1 / Origine taxonomique}

Le premier critère qui intéresse les producteurs de poissons de bouche est le potentiel de croissance lié à un bon indice de conversion alimentaire. Dans presque tous les cas, l'espèce ou la souche (population) de poisson à domestiquer a déjà un intérêt halieutique reconnu. C'est donc sur la base de la taille (moyenne ou maximale) qu'une espèce d'origine sauvage est jugée digne d'intérêt, qu'elle soit d'origine marine (environ 14000 espèces) ou dulçaquicole (environ 10000 espèces). Même si la relation "taille maximale-vitesse de croissance" n'est pas absolue, la taille reste en moyenne un bon indicateur des potentialités de croissance de l'espèce candidate.

Si certaines familles de téléostéens sont plutôt constituées d'espèces de grande taille (Thonidés, Acipenseridés, Ariidés, Pimelodidés) ou de petite taille (Poecilidés, Trichomycteridés, Callichthyidés), beaucoup d'entre elles présentent une hétérogénéité de taille très importante (Cyprinidae, Characidae, Loricaridés, Cichlidés). Cette variabilité se réduit au sein d'un même genre, mais cette règle subit de nombreuses exceptions qui seraient, au moins pour partie, liées à la définition "artificielle" du périmètre des genres.

Au sein même d'une espèce, on observe des différences notables de taille entre populations. Ces différences peuvent être dues aux contraintes imposées par le milieu (cas des saumons landlock qui ne peuvent plus migrer et dont la taille s'en trouve très réduite) ou à l'absence d'une espèce proche en compétition pour la même niche écologique (taille maximale accrue des populations d'Hoplias malabaricus ou de Myleus ternetzi dans les cours d'eau où respectivement Hoplias aimara et Myleus rubripinnis sont absents ; Planquette et al 1996). Toutefois, il est difficile de distinguer entre une divergence génétique (évolution) des populations et une disponibilité alimentaire variable. Seul un élevage comparatif en milieux contrôlés identiques permettrait de conclure (Vandeputte et Launey 2004).

A partir des seules informations des traits de vie et des caractéristiques écologiques, il apparaît difficile de trouver, a priori, des règles de choix d'une souche de départ qui aurait des potentialités de croissance plus importantes qu'une autre. Cependant, la grande plasticité de la croissance observée laisse présager de gains importants de croissance qui pourraient être obtenus suite à une pression de sélection orientée (ce qui est confirmé par les différents exemples obtenus en aquaculture sur la truite ou la carpe). 


\section{2 / Influence des facteurs environnementaux}

Chez les poissons, contrairement aux mammifères et aux oiseaux chez lesquels la néomyogenèse est extrêmement réduite après la naissance, le nombre de fibres musculaires, dont dépend en grande partie la taille maximale de l'animal n'est pas toujours déterminé à l'éclosion (Stickland et al 1988, Rowlerson et Veggetti 2001). Ainsi, chez les espèces de grande taille comme la truite, les cellules musculaires souches (cellules satellites) continuent à se multiplier (hyperplasie) bien au-delà du premier cycle de reproduction, alors qu'une espèce de petite taille comme le poisson-zèbre a une croissance post-éclosion essentiellement due à l'augmentation de la taille des fibres musculaires (hypertrophie). Mais si la croissance musculaire, qu'elle soit de type hyperplasique ou hypertrophique, est directement liée à l'énergie disponible pour favoriser l'accrétion protéique, sa vitesse n'est pas linéaire : il existe en effet des rythmes endogènes de croissance. Ainsi, il a été montré, chez le tilapia par exemple (Massou et al 2002), un dépôt journalier de stries d'accroissement de l'otolithe, et, chez la truite, un rythme de croissance mensuel qui, cependant, pourrait être lié au rythme lunaire (Noël et Le Bail 1997). Toutefois, les différentes espèces de poissons présentent très souvent des rythmes saisonniers qui sont la résultante de l'influence de la disponibilité alimentaire, de la qualité chimique de l'eau (particulièrement chez les espèces tropicales d'eau douce), de la température ambiante et de la photopériode (surtout pour les espèces de climat tempéré).

Les poissons sont des poïkilothermes obligatoires, parfois sensibles à des écarts de température de $0,5^{\circ} \mathrm{C}$, dont les préférences thermiques varient, selon les espèces, entre $-2,5^{\circ} \mathrm{C}$ et $44^{\circ} \mathrm{C}$ (Elliot 1981). Chaque espèce ne peut survivre qu'au sein d'une plage de températures bien définie, et la plage des températures optimales pour la croissance est encore plus étroite (autour de $16^{\circ} \mathrm{C}$ pour la truite et $25^{\circ} \mathrm{C}$ pour la carpe). Il n'est pas exclu que cet optimum puisse varier suivant les populations, comme le suggère la sélection réussie d'une souche de carpe résistante au froid (Vandeputte et Launey 2004). Au-delà, la température diminue la croissance et peut devenir un facteur de stress.

Dans une gamme de température dite physiologique, où la croissance peut s'exprimer, l'élévation de la température augmente la prise alimentaire parallèlement au métabolisme basal (Brett et Groves 1979). Il ne faut pas négliger le fait que la diminution du métabolisme général due aux faibles températures puisse, comme chez les mammifères, ralentir le vieillissement et augmenter la longévité ainsi que la taille maximale des individus (Le Bourg 1999).

Des effets plus spécifiques que la simple prise alimentaire ont pu être démontrés. Ainsi, durant le développement embryonnaire, de faibles températures peuvent induire une augmentation du nombre de fibres musculaires (Stickland et al 1988, Usher et al 1994, Nathanailides et al 1995) et du nombre de vertèbres (Kwain 1975) à l'éclosion, augmentant ainsi le potentiel de croissance ultérieure. Par ailleurs, les concentrations d'hormones comme la thyroxine et l'hormone de croissance $(\mathrm{GH})$, connues pour favoriser la prise alimentaire (Le Bail et Bouf 1997), sont augmentées par la température (Gabillard et al 2003 et 2004) induisant, en plus d'une augmentation du métabolisme général, une amélioration du coefficient de conversion alimentaire (Donaldson et al 1979).

Les effets de la température sur la croissance sont donc multiples et parfois contradictoires. Le choix d'une espèce ou d'une souche à domestiquer doit être fait en fonction du système d'élevage prévu. Une fois les conditions thermiques fixées, les critères de sélection pour la croissance doivent, suivant les objectifs visés, prendre aussi en compte la longévité, le taux de conversion alimentaire (en s'appuyant ou non sur les taux hormonaux plasmatiques) sans oublier la conformation générale du corps et la qualité de la chair.

Les conditions d'éclairement des poissons sont certainement parmi les conditions les plus difficiles à appréhender dans le milieu naturel (luminosité fluctuante, hétérogénéité de l'éclairement du milieu, préférendum ou évitement des poissons...). Le spectre lumineux est très certainement important pour les conditions de vie des poissons, mais les informations fiables sur leurs effets manquent (difficulté méthodologique à expérimenter), ce qui a conduit les pisciculteurs à utiliser empiriquement des lampes mimant le spectre solaire dans leurs élevages d'intérieur.

En revanche, les effets de l'intensité lumineuse et de la photopériode sur le développement et la croissance sont mieux documentés (Bøuf et Le Bail 1999). Généralement, une intensité minimale est nécessaire pour un développement harmonieux et une croissance normale des juvéniles en permettant l'accès à l'alimentation, au moins chez les espèces diurnes. Chez les animaux plus gros, l'intensité lumineuse ne semble pas avoir d'influence déterminante.

La photopériode agit souvent comme un synchroniseur des rythmes endogènes de l'animal. Les photopériodes longues peuvent favoriser la croissance, comme chez la truite (Maisse et al 1998) et le saumon atlantique qui y est particulièrement sensible (Bœuf et Le Bail 1999). De fait, les salmoniculteurs du nord de l'Europe utilisent des photopériodes longues ou un éclairage continu pour compenser les basses températures en période hivernale. Mais ce type d'approche n'est pas généralisable car de nombreuses espèces n'y sont pas sensibles. C'est donc une démarche au cas par cas qu'il faut emprunter pour déterminer les photopériodes optimales pour les espèces en cours de domestication.

Les milieux aquatiques peuvent présenter des salinités (ici nous ne parlerons que du 
$\mathrm{NaCl}$ ) très variables allant de 0 psu en eau douce jusqu'à des salinités extrêmes > 100 psu (mare hypersaline) en passant par 35 psu qui est la valeur moyenne de l'eau de mer. Si pour beaucoup d'espèces, le passage dans un milieu dont la salinité est éloignée de celle de son milieu "physiologique" conduit à un ralentissement ou un blocage de la croissance, voire à la mort, d'autres supportent de telles variations qui peuvent être favorables à la croissance corporelle (Bœuf et Payan 2001). C'est le cas d'espèces marines comme certains Scianidés (Micropogonias), Sparidés (Sparus), Mugilidés (Mugil, Chelon), comme la morue (Gadus morhua), le turbot (Scophthalmus maximus) ou le bar (Dicentrarchus labrax) ou d'espèces dulçaquicoles comme les tilapias (Oreochromis), Cyprinidés (Cyprinus, Ctenopharyngodon) et le milkfish (Chanos chanos). Chez certaines de ces espèces, le chlorure de sodium agirait sur la croissance via la prise alimentaire (cas du turbot), voire via l'indice de transformation alimentaire (cas de la morue).

Les nombreuses espèces euryhalines, au cours de leur changement de milieu (eau douce vers les eaux saumâtres ou marines et réciproquement), doivent s'adapter en osmorégulant pour maintenir l'homéostasie de leur milieu intérieur. Le maintien de cet équilibre a un coût énergétique qui peut affecter les performances de croissance. Théoriquement, le moindre coût énergétique devrait être obtenu lorsque la pression osmotique du milieu extérieur est proche de celle du milieu intérieur de l'animal. Ce coût est estimé, selon les auteurs, entre 10 et plus de $50 \%$ de métabolisme basal. De fait, on observe des croissances optimisées entre 8 et 16 psu (Bouf et Payan 2001) chez plusieurs espèces. Mais la plupart du temps, l'optimum de croissance est obtenu pour les valeurs supérieures ou inférieures à ces valeurs.

Ces espèces euryhalines ont mis en place des stratégies de changement de milieu dont elles sont devenues, a posteriori, plus ou moins tributaires (cas des déplacements dans le gradient de salinité des estuaires). Dans certains cas, ces stratégies sont devenues obligatoires (saumon, anguille) sous peine de perturber profondément les autres fonctions physiologiques. Ainsi, le maintien en eau douce du saumon atlantique au stade parr (type landlock) conduit à des mortalités très importantes et à une croissance réduite. Les saumons et, dans une moindre mesure, les truites se préparent à leur migration catadrome en smoltifiant (allongement du corps, prise d'une livrée argentée, préparation à l'osmorégulation en milieu salin). Ces changements s'accompagnent d'une accélération remarquable de la croissance corporelle (Thorpe et al 1982, Baglinière et Maisse 1985) qui est due à l'augmentation des concentrations sanguines de l'hormone de croissance (GH), d'IGFs et d'hormones thyroïdiennes (Bouf et al 1989), hormones également impliquées dans la prise alimentaire et l'osmorégulation. Ces augmentations sont plus précoces quand les individus proviennent de populations plus éloignées de la mer (Bœuf et Le Bail 1990). D'où la nécessité de choisir, pour une même espèce, les souches les plus adaptables au milieu d'élevage envisagé ou bien de cerner les différentes phases des cycles de migration (cas de nombreuses espèces) pour mieux adapter les conditions d'élevage aux exigences de l'espèce.

Parmi les autres facteurs d'élevage pouvant influencer la croissance, la densité joue un rôle particulier, parfois paradoxal. Les espèces grégaires acceptent généralement des densités d'élevage relativement élevées. Cependant, une densité trop élevée peut conduire à des inhibitions de croissance (Brett 1979). Celles-ci sont souvent reliées à l'accroissement des interactions entre individus et au stress qu'elles génèrent : ainsi, chez le pagre (Pagrus pagrus), une densité trop élevée réduit la distance entre individus en deçà de la distance minimale acceptable, conduisant à des agressions répétées entre individus avec morsures, perte d'œil et mort. A l'opposé, les salmonidés, connus pour être très territoriaux dans le milieu naturel (Heland 1991), doivent subir des densités très supérieures à celles observées dans le milieu naturel pour "diluer" l'intensité et la fréquence des attaques des dominants sur leurs congénères et ainsi favoriser la croissance (Brett 1979). Il est donc difficile de prédire quelle sera l'aptitude à la domestication d'une espèce sur la seule observation de son comportement dans son milieu naturel.

Des analyses et des conclusions similaires pourraient être avancées pour les autres facteurs environnementaux connus pour agir sur la croissance : oxygène, autres paramètres chimiques de l'eau, substrat, structure des bassins d'élevage, taille des animaux.

Souvent, lors de la première phase de domestication, les conditions d'élevage sont empiriquement choisies proches des conditions de vie de l'animal dans le milieu naturel. Cependant, ces conditions sont rarement compatibles avec la rentabilité économique et les systèmes d'élevage envisagés, surtout lorsqu'ils sont intensifs. Pour réussir la phase de domestication, il faut donc rechercher des compromis. La plasticité de la croissance en réponse aux facteurs environnementaux laisse augurer des potentialités d'adaptation des espèces à de nouveaux environnements, en imposant une certaine pression de sélection dans un cadre se rapprochant peu à peu du système d'élevage recherché. De fait, les premiers gains de croissance obtenus sont souvent plus liés à une diminution de la réponse à des situations de stress (résistance aux maladies, baisse des interactions de dominance, adaptabilité à un nouvel environnement ...) et à une augmentation de la prise alimentaire qu'à une augmentation des potentialités de croissance proprement dites (Vandeputte et Launey 2004). Cependant, il faudra veiller à ce que l'optimisation des conditions d'élevage en vue d'une croissance maximale ne s'oppose pas au déroulement optimal d'autres fonctions comme la reproduction. En effet, chez la truite, l'optimum de croissance se situe vers $16^{\circ} \mathrm{C}$ alors que la qualité des oufs nécessite une température inférieure à $12^{\circ} \mathrm{C}$ et chez la carpe, une période de vernalisation 
est obligatoire pour permettre le bon déroulement de la gamétogenèse.

\section{3 / Interactions entre fonctions}

Les variations saisonnières de croissance, induites par les fluctuations de certains facteurs externes, peuvent être amplifiées ou réduites par la mise en place et le déroulement d'autres fonctions physiologiques.

La fin du développement embryonnaire survient durant la saison la plus favorable à la survie et à la croissance des alevins (abondance de plancton : saison des pluies en zones tropicales, printemps en zones tempérées) qui synchronisera le démarrage et le déroulement du cycle de reproduction y compris la ponte. L'impact de la fonction de reproduction sur la croissance corporelle peut se résumer à deux phases bien identifiées chez les salmonidés (Le Bail 1988). La première phase inclut le démarrage du cycle (puberté lorsqu'il s'agit du premier cycle) et la phase lente de développement de la gonade (prévitellogenèse jusqu'au début de la vitellogenèse exogène). Durant cette période, la croissance corporelle est souvent accélérée (comparée à celle de témoins immatures) et l'animal accumule les graisses périviscérales et musculaires. Durant la seconde phase, qui couvre la période de fort accroissement de la gonade (vitellogenèse exogène très active) jusqu'à la ponte, on observe un ralentissement ou un arrêt de la croissance corporelle, voire une diminution du poids corporel, souvent dû à une diminution ou à un arrêt de la prise alimentaire, soit avant la période de reproduction (Salmonidés), soit durant la période d'incubation des œufs (Cichlidés, Ariidés ...).

Ces variations de croissance corporelle sont la résultante de deux phénomènes : 1) la stimulation de la croissance par certaines hormones anabolisantes (stéroïdes sexuels à faible concentration, thyroxine, hormone de croissance) durant la première phase de la gamétogénèse (Le Gac et al 1993) ; 2) le ralentissement de la croissance corporelledurant la seconde phase de la gamétogénèse dû à la réorientation massive de l'énergie absorbée (ou stockée) vers le développement des gonades (constitution des réserves vitelline chez la femelle ou du sperme chez les mâles) couplée à un effet inhibiteur des fortes concentrations des stéroïdes sexuels (Le Bail 1988, Toguyeni et al 1996, Tacon et al 2000, Massou et al 2004). Les différences de croissance qui peuvent être observées entre sexes (favorables aux mâles chez le tilapia et l'atipa, le mulet ... ou aux femelles chez la truite, l'alose, le barbeau, la perche ...) sont, pour partie, expliquées par ces mécanismes. En effet, lorsque le démarrage du cycle reproducteur est plus précoce pour un sexe (1 à 2 ans chez de nombreuses espèces), il en résulte un différentiel de croissance corporelle, souvent négatif, avec l'autre sexe qui mature plus tardivement.

D'autre part, l'entrée en puberté dépend plus de la taille de l'animal que de son âge (Le
Bail 1988). Généralement et globalement, la puberté survient chez les individus dont le poids corporel atteint entre le quart et le tiers du poids maximal de l'espèce ou de la population étudiée, les populations à croissance corporelle rapide étant matures plus précocement.

Du point de vue aquacole, il y a donc contradiction à vouloir obtenir, d'une part, des poissons maturant tardivement pour éviter les ralentissements de croissance corporelle et la dégradation de la qualité de la chair (perte des lipides musculaires) et, d'autre part, l'intérêt qu'apportent des souches à puberté précoce pour accélérer l'amélioration génétique en raccourcissant la durée entre générations. Ce problème est accentué par la recherche d'une croissance corporelle rapide (obtenue par sélection ou par amélioration des conditions zootechniques) qui accélère l'entrée en puberté, même s'il est possible de dissocier partiellement ce lien. Face à ces difficultés, les techniques de stérilisation et de monosexage (telles qu'elles sont pratiquées chez les Salmonidés) doivent être envisagées lors du choix des espèces candidates à la domestication, et testées dès les premiers cycle de vie pour vérifier la faisabilité d'une telle approche.

La fécondité des femelles (qui est liée à la taille de l'animal) et la qualité des gamètes peuvent être très perturbées (en nombre comme en taille) par les conditions de croissance corporelle durant les premières phases de la période post-larvaire, peu de temps avant l'entrée en puberté et durant toute la période de la gamétogenèse (Le Bail 1988). Ces fortes interactions entre alimentation (qualité et quantité), croissance corporelle et reproduction doivent être prises en compte pour favoriser de bonnes conditions de reproduction.

\section{Conclusion}

Si établir une typologie fonctionnelle des espèces aux premiers stades de développement (larve, post-larve, juvénile) semble prometteur pour mieux appréhender la domestication de nouvelles espèces, de futures investigations devront confirmer la pertinence de la démarche. La très grande plasticité de croissance (variabilité intra et interspécifique) qui existe chez les poissons ne permet pas, à ce stade de la réflexion, un regroupement d'espèces pour cette fonction biologique. En fait, lors de la domestication d'une nouvelle espèce, le changement d'environnement va affecter sa plasticité de croissance sous l'influence de la pression de sélection. De ce fait, il est indispensable que le milieu de sélection soit le plus proche possible du futur milieu d'élevage. Il convient de noter que la vitesse de croissance reste un excellent indicateur de l'acclimatation des poissons aux nouvelles conditions d'élevage. La sélection d'individus, ciblée sur leurs performances de croissance, nécessitera une évaluation des effets secondaires sur les autres fonctions biologiques, notamment la reproduction. 


\section{Références}

Babiak I., Mandiki S.N.M., Ratsinjomanana K., Kestemont P., 2004. Initial weight and its variation in postlarval Eurasian perch affect quantitative characteristics of juvenile cohorts under controlled conditions. Aquaculture, 234, 263-276.

Bagenal T.B., 1971. The interrelation of the size of fish eggs, the date of spawning and the production cycle. J. Fish Biol., 3, 307-319.

Baglinière J.L., Maisse G., 1985. Precocious maturation and smoltification in wild Atlantic salmon in the Armorican massif, France. Aquaculture, 45, 249-263.

Baras E., 1998. Bases biologiques du cannibalisme chez les poissons. Cah. Ethol Appl., 18, 53-98.

Baras E., Jobling M., 2002. Dynamics of intracohort cannibalism in cultured fish. Aquac. Res., 33, 461-479.

Bouf G., Le Bail P.-Y., 1990. Growth hormone and thyroid hormone levels during smolting in different populations of atlantic salmon. Progr. Comp.Endocrinol., 193-197.

Boeuf G., Le Bail P.-Y., 1999. Does light have an influence on fish growth? Aquaculture, 177, 129-152

Bœuf G., Payan P., 2001. How should salinity influence fish growth? Comp. Biochem. Physiol. C, 130, 411-423.

Bœuf G., Le Bail P.-Y., Prunet P., 1989. Growth hormone and thyroid hormones during atlantic salmon, Salmo salar L., smolting, and after transfert to seawater. Aquaculture, $82,257-268$.

Bone Q., Marshall N.B., Blaxter J.H.S., 1996. Biologie of Fishes. Chapman and Hall, $2^{\text {nd }}$ Edition, 332 p.

Brett J.R., 1979. Environmental factors and growth. In: Hoar W.S., Randall D.J. and Brett J.R. (eds), Bioenergetics and Growth, 599-675. Academic Press, New York.

Brett J.R., Groves T.D.D., 1979. Physiological energetics. In: Hoar W.S., Randall D.J. and Brett J.R. (eds), Bioenergetics and Growth, 280-244. Academic Press, New York.

Cahu C., 2004. Domestication et fonction nutrition chez les poissons. INRA Prod. Anim., 17, 205-210.

Conover D.O., 1992. Seasonality and scheduling of life history at different lattitudes. J. Fish Biol. 41 (suppl. B), 161-178.

Dabrowski K., Culver D.A., Brooks C.L., Voss A.C., Binkowski F.P., Yeo S.E., Balogun A.M., 1993. Biochemica aspects of the early life history of yellow perch (Perca flavescens). In: S.J. Kaushik and P. Luquet (eds), Fish Nutrition in Practice, 531-539. INRA, 4th Symposium in Fish Nutrition and Feeding, Biarritz, June, 24-27, France.

Donaldson E.M., Fagerlund U.H.M., Higgs D.A., Mc Bride J.R., 1979. In: Hoar W.S., Randal D.J. and Brett J.R. (eds), Fish Physiologie, Vol VII, 334-338. Academic Press, New York.

Elliot J.M., 1981. Some aspects of thermal stress on freshwater teleosts. In: Pickering A. (ed), Stress in Fish, 209245. Academic Press, London

Fontaine P., 2004. Premiers résultats en Perciculture hors-sol chez Lucas Perche. Aquafilia, 2, 10-14.

Gabillard J.-C., Weil C., Rescan P.Y., Navarro I., Gutierrez J., Le Bail P.-Y., 2003. Environmental temperature increases plasma GH levels independently of the nutritional status in rainbow trout (Oncorhynchus mykiss). Gen. Comp. Endocrinol., 133, 17-26.

Gabillard J.-C., Weil C., Rescan P.Y., Navarro I., Gutierrez J., Le Bail P.-Y., 2004. Does the GH/IGF system mediate the effect of temperature on fish growth ? Cybium, sous presse.

Hecht T., Pienaar A.G., 1993. A review of cannibalism and its implication in fish larviculture. J. World Aquac. 24, 246-261.

Heland M., 1991. Organisation sociale et territorialité chez la truite commune immature au cours de l'ontogénèse. In : Baglinière J.L. et Maisse G. (eds), La truite : biologie et écologie, 121-149. INRA éditions, Paris.
Huston M.A., DeAngelis D.L., 1987. Size bimodality in monospecific populations: a critical review of potential mechanisms. Am Nat. 129, 678-707.

Jourdan S., 1999. Influence de facteurs abiotiques, la photopériode et l'intensité lumieuse, sur la survie et la croissance de larves, post-larves et juvéniles de perche Perca fluviatilis L.. Thèse de l'INPL, Nancy, $147 \mathrm{p}$.

Kamler E., 1992. Early life history of fish. An energetics approach. Kluwer Academic publishers, 1st Edition, $267 \mathrm{p}$

Keith P., Le Bail P.-Y, Planquette P., 2000. Atlas des poissons d'eau douce de Guyane. Tome 2, fascicule I : Batrachoidiformes, Mugiliformes, Beloniformes Cyprinodontiformes, Synbranchiformes, Perciformes, Pleuronestiformes, Tetraodontiformes. Editions MNHN/SPN, collection Patrimoines naturels, vol 43, 286 p.

Kestemont P., Mélard, C., 2000. Chap. 11: Aquaculture In: J.F. Craig (ed), Percids Fish. Systematics, Ecology and Exploitation, 191-224. Blackwell Sciences, Fish and Aquatic Resources Series 3.

Kestemont P., Jourdan S., Houbart M., Mélard C., Paspatis M., Fontaine P., Cuvier-Peres A., Kentouri M., Baras E., 2003. Size heterogeneity, cannibalism and competition in cultured predatory fish larvae: biotic and abiotic influences. Aquaculture, 227, 333-356.

Kwain W., 1975. Embryonic development, early growth and meristic variation in rainbow trout (Salmo gairdneri) exposed to combinations of light intensity and temperature. J. Fish. Res. Bd. Canada, 32, 397-402.

Le Bail P.-Y., 1988. Growth-reproduction interaction in salmonids. In: Zohar Y. and Breton (eds), Reproduction in fish, Basic and applied aspects in endocrinology and genetics, 91-108. INRA Editions, Paris, collection Les colloques de l'INRA $n^{\circ} 44$.

Le Bail P.-Y., Bouf G., 1997. What hormones may regulate food intake in fish ? Aquat. Living Resour., 10, 371-379.

Le Bail P.-Y, Keith P., Planquette P., 2000. Atlas des poissons d'eau douce de Guyane. Tome 2, fascicule II Siluriformes. Editions MNHN/SPN, collection Patrimoines naturels, vol 43, $307 \mathrm{p}$.

Le Bourg E., 1999. Le bestiaire de la gérontologie expérimentale. La Recherche, 322, 39-43.

Le Gac F., Blaise O., Fostier A., Le Bail P.Y., Loir M. Mourot B., Weil C., 1993. Growth hormone (GH) and reproduction: a review. Fish Physiol. Biochem., 11, 219-232.

Maisse G., Noël O., Breton B., Goardon L., P.-Y. Le Bail, Mourot $B, 1998$. Influence dune période d'éclairement continu sur la première maturation sexuelle de la truite arcen-ciel (Oncorhynchus mykiss). Bull. Fr. Pêche Piscic. $350-351,265-264$.

Mandiki S.N.M., Blanchard G., Mélard C., Koskela J., Kucharczyk D., Fontaine P., Kestemont P., 2004. Effects of geographic origin on growth and food intake in Eurasian perch (Perca fluviatilis L.) juveniles under intensive culture conditions. Aquaculture, 229, 117-128.

Massou A.M., Panfili J., Laë R., Baroiller J.F., Mikolasek O., Fontenelle G., Le Bail P.-Y., 2002. Effects of different food restrictions on somatics and otolith growth in Nile tilapia reared under controlled conditions. J. Fish Biol., 60 1093-1104.

Massou A.M., Panfili J., Le Bail P.-Y., Laë R., Mikolasek O., Fontenelle G., Baroiller J.F., 2004. Evidence of perturbations induced by reproduction on somatic growth and microincrement deposition in Oreochromis niloticus otoliths. J. Fish Biol., 64, 380-398.

Naas K., Huse I., Iglesias J., 1996. Illumination in first feeding tanks for marine fish larvae. Aquacult. Eng., 15, 291300 .

Nathanailides C., Lopez-Albors O., Stickland N.C., 1995. Influence of prehatch temperature on the development of muscle cellularity in posthatch Atlantic salmon (Salmo salar). Can. J. Fish Aquat. Sci., 52, 675-680. 
Nishimura K., Hoshino N., 1999. Evolution of cannibalism in the larval stage of pelagic fish. Evolutionary Ecology, 13, 191-209.

Noël O., Le Bail P.-Y., 1997. Does cyclicity of growth rate in rainbow trout exist? J. Fish Biol., 51, 634-642.

Planquette P., Keith P., Le Bail P.-Y,.1996. Atlas des poissons d'eau douce de Guyane (Tome 1). Collection du Patrimoine Naturel, vol 22, Edition IEGB-MNHN, INRA, CSP, Min. Env., Paris, 429 p.

Ricker W.E., 1979. Growth rates and models. In: W.S. Hoar, D.J. Randall and J.R. Brett (eds), Fish Physiology, Vol. VIII, 677-743. Academic Press, London.

Rieger P.W., Summerfelt, R.C., 1997. The influence of turbidity on larval walleye, Stizostedion vitreum, behavior and development in tank culture. Aquaculture, 159, 19-32.

Rowlerson A., Veggetti A., 2001. Cellular mechanisms of post-embryonic muscle growth in aquaculture species. In: I.A. Johnston (ed), Fish Physiology, Vol. 18: Muscle developpment and growth, 103-140. Academic Press, London.

Schultz E.T., Reynolds K.E., Conover D.O., 1996. Countergradient variation in growth among newly hatched Fundulus heteroclitus: geographic differences revealed by common-environment experiments. Funct. Ecol., 10, 366374 .
Stickland N.C., White R.N., Mescall P.E., Crook A.R. Thorpe J.E., 1988. The effect of temperature on myogenesis in embryonic development of the Atlantic salmon (Salmo salar L.). Anat. Embryol. (Berl), 178, 253-257.

Tacon P., Baroiller J.F., Le Bail P.-Y., Prunet P., Jalabert B. 2000. Effect of egg deprivation on sex steroïds, gonadotropin, prolactin, and growth hormone profiles during the reproductive cycle of the mouthbrooding cichlid fish Oreochromis niloticus. Gen. Comp. Endocrinol. 117, 54-65.

Thorpe J.E., Talbot C., Villarreal C., 1982. Bimodality of growth and smolting in atlantic salmon, Salmo salar L. Aquaculture, 28, 123-132.

Toguyeni A., Baroiller J.F., Fostier A., Le Bail P.-Y., Kühn E.R., Mol K.A., Fauconneau B., 1996. Consequences of food restriction on short-term growth variation and on plasma circulating hormones in Oreochromis niloticus in relation to sex. Gen. Comp. Endocrinol., 103, 167-175.

Usher M.L., Stickland N.C., Thorpe J.E., 1994. Muscle development in Atlantic salmon (Salmo salar) embryos and the effect of temperature on muscle celullarity. J. Fish Biol., 44, 237-242.

Vandeputte M., Launey S., 2004. Quelle gestion génétique de la domestication chez les poissons? INRA Prod. Anim. $17,237-242$.

Wotton R.J., 1999. Ecology of teleost fishes. Kluwer Academic Publishers, 2nd Edition, 386 p.

\begin{abstract}

\section{Domestication and growth in fish.}

Studying two successive phases of fish production cycle, larval rearing and growing phase, fish growth patterns were analysed to perform a functional typology. For all developmental stages, high growth heterogeneities (intra and inter-specific) were observed. They were explained by the effects of intrinsic and external factors (biotic and abiotic). For the growing phase, this high phenotypic variability does not allow to sort out species according to their growing pattern. This

effect is partly due to strong interactions of growth with other physiological functions (e.g. reproduction). Growing pattern does not seem to be discriminative. For larval rearing (larval, post-larval and juvenile stages), growth heterogeneity and cannibalism could be related to the living strategies of fish species.

FONTAINE P., LE BAIL P.-Y., 2004. Domestication et croissance chez les poissons. INRA Prod. Anim., 17, 217-225.
\end{abstract}


